\title{
Reproducibility of histopathological findings in experimental pathology of the mouse: a sorry tail
}

\author{
Jerrold M Ward ${ }^{1}$, Paul N Schofield ${ }^{2,3}$ \& John P Sundberg ${ }^{3}$
}

Reproducibility of in vivo research using the mouse as a model organism depends on many factors, including experimental design, strain or stock, experimental protocols, and methods of data evaluation. Gross and histopathology are often the endpoints of such research and there is increasing concern about the accuracy and reproducibility of diagnoses in the literature. To reproduce histopathological results, the pathology protocol, including necropsy methods and slide preparation, should be followed by interpretation of the slides by a pathologist familiar with reading mouse slides and familiar with the consensus medical nomenclature used in mouse pathology. Likewise, it is important that pathologists are consulted as reviewers of manuscripts where histopathology is a key part of the investigation. The absence of pathology expertise in planning, executing and reviewing in vivo research using mice leads to questionable pathology-based findings and conclusions from studies, even in high-impact journals. We discuss the various aspects of this problem, give some examples from the literature and suggest solutions.

Histopathological descriptions of the frequency and nature of lesions and disease entities are very often the endpoints in biomedical research conducted in model organisms such as the mouse. In contrast with clinical pathology, where endpoints are usually assessed using biochemical and molecular assays, histopathological assessment, despite using molecular markers and imaging as adjunct qualitative and quantitative techniques, is highly dependent on the individual expertise of trained expert pathologists. Pathologists must not only recognize lesions, but also have knowledge of the background diseases of the mice and understand the meaning of the pattern of disease in the whole mouse ${ }^{1-4}$. Reproducibility of histopathological endpoints therefore depends on the implementation of a common standardized vocabulary, competent work-up and an in-depth knowledge of the mouse strains under investigation so that, for example, background lesions are not mistaken for those that are experimentally induced. Such knowledge is critical in the design of experiments, as well as in understanding the effects of husbandry, the microbiome and diet on the interpretation of results ${ }^{2,5}$.

In recent years, funding agencies and scientific communities alike have expressed increasing concern about the lack of reproducibility of experiments in the biomedical domain. Attention was initially drawn to this issue by pharmaceutical companies, which rely on preclinical, precompetitive research for drug development pipelines $^{6-8}$. Identification of this problem has been followed by an outpouring of concern from funding agencies such as the US National Institutes of Health ${ }^{9-11}$ and, to an extent, journals and professional bodies ${ }^{12-17}$.
Although much attention has been paid to the reproducibility of molecular assays, in vitro (cell culture) assays and the inappropriate application of statistical methods, only recently have the issues surrounding reproducibility in animal experimentation been discussed in depth ${ }^{13}$. Many of these discussions have concerned husbandry and the effect of diet and microbiome on experimental outcomes ${ }^{18-20}$, particularly in neuroscience ${ }^{14}$. However, recent papers have addressed the problem of what a sound histopathological investigation should look like, how to use knowledge of pathology in experimental design, based on the ARRIVE and related guidelines, and the confounding effect of the environment and the gut and skin microbiomes ${ }^{5}$.

Here we address some of the issues that affect reproducibility of histopathological findings: a lack of pathology expertise, in author lists and in peer review; poor standards of reporting, as illustrated by common errors seen in papers, and inconsistent pathology nomenclature; and availability of primary data, without which it is impossible to assess a paper without attempting a complete experimental replication ${ }^{21-23}$. Notably, we emphasize that if pathologists are not involved in designing mouse experiments and interpreting lesions, the accuracy of the diagnoses reported and the conclusions drawn may be questionable.

\section{The importance of pathologists}

Pathology is a medical specialty that requires years of training, experience and board certification as a minimum. Although pathology has many sub-disciplines, such as mouse pathology,

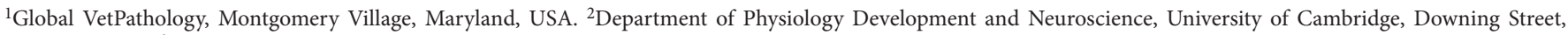
Cambridge, UK. ${ }^{3}$ The Jackson Laboratory, Bar Harbor, Maine, USA. Correspondence should be addressed to J.M.W. (veterinarypathology@gmail.com). 


\author{
Table 1 | Evidence of questionable pathology interpretation in \\ publications \\ Figure legends do not accurately reflect what is in the figure \\ Figure legends do not describe anything in the figure \\ Lack of complete or appropriate necropsies and histopathology \\ Misidentification of normal organs and tissues as lesions \\ Diagnoses of non-neoplastic lesions as neoplasms \\ Diagnoses of tumors with unconventional terminology \\ Reporting of benign lesions as malignant \\ Reporting of inflammatory lesions as neoplasms \\ Reporting of novel lesions incorrectly \\ Use of incorrect (accepted) terminology/diagnoses
}

a general pathologist is much more of an expert than a nonpathologist, and is often sufficient to provide substantial benefit to an animal research study ${ }^{24}$. However, investigators often do not have enough funds to pay for research pathology services and/or believe that they can perform histopathology interpretations themselves. A lack of pathology expertise by investigators leads to inaccurate histopathological descriptions of lesions and often missed, or spurious reporting of, pathological findings in publications. Absence of a pathologist may be noticed in the figure legends, which often do not describe the lesions displayed, or, in some rare cases, in images that are replicated in various orientations for different lesions of mice ${ }^{25}$. In some cases, a pathologist was not involved in late of final edits or did not review the galley proofs of an accepted manuscript ${ }^{26}$, leading to a substantial error in reporting.

In addition to accurate interpretation of data, pathologists are important for ensuring that proper nomenclature is used when reporting on results. The use of generally accepted pathology nomenclature for unexpected and new findings leads to publications that can be interpreted by readers, including other pathologists. Rodent pathology terminology often mirrors that used for humans, but there are differences. The pathology of genetically engineered mice often requires interpretation of new findings, as each mouse may have unique lesions that have not been previously reported, especially when the study is the first for a new gene knockout or treatment. A classic example is the relatively common lesion in mouse hearts that pathologists diagnose as epicardial and myocardial mineralization and fibrosis, but non-pathologists often call dystrophic cardiac calcinosis or a variety of other names ${ }^{27-31}$. Investigators without pathology backgrounds often over-interpret their research findings, with the temptation being to fit results to their hypotheses. Over-diagnosis of lesions as malignant when they may be, in fact, benign, hyperplastic or even normal is a common problem. This latter point emphasizes the value of knowing the anatomical differences between the species.

\section{Some examples of errors seen in reported histopathological diagnoses}

In addition to incomplete reporting of the experimental design, including the pathology protocol, there are common questionable

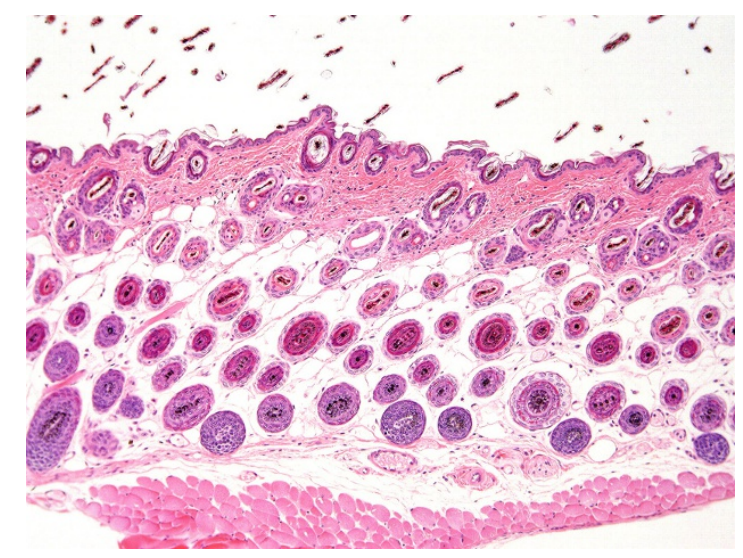

FIGURE 1 | Skin of mouse in anagen with abundant hair follicles. This normal stage of the cell cycle has been reported to be a marked increase in hair follicles, when in fact it is an orientation artifact. Hematoxylin and eosin stain, $10 \times$ magnification.

diagnoses that can be found in published results (Table 1) ${ }^{32-34}$. Often, the figure legends do not describe what is illustrated by the figure, normal tissues are misidentified as lesions, non-neoplastic lesions are reported as cancer or benign lesions are diagnosed as malignant neoplasms. In addition, inflammatory lesions may be described as neoplasms, benign or malignant.

Our interpretations of histopathology figures in published reports, which we will give as examples, are based solely on our interpretation of what was present in the published figures and are not based on microscopic slide review, which may reveal different findings than what is in the published figures. Often, the published histopathology figures are small, and they can lose resolution when enlarged, to the point of being uninterpretable. One of many approaches to this problem is to post additional digital images at a variety of magnifications or whole-slide images as supplemental data. Images could also be posted on public websites such the Mouse Tumor Biology Database ${ }^{35,36}$, Gene Expression Database ${ }^{37}$, Pathbase ${ }^{38,39}$ and many others ${ }^{5}$.

To evaluate any organs, a clear understanding of the normal anatomy is absolutely necessary to recognize any type of change, be it disease or just subtle changes in normal physiology. When evaluating the skin, the normal hair cycle is a commonly reported source of misinterpretation. All hair follicles regularly go through anagen, the normal growth phase; catagen, the transition stage to telogen, the long-term resting stage; to exogen, when the old hair shaft is lost. This process then starts over again and is repeated throughout life. The cycle varies by hair type (vibrissae cycle is different compared with body hair) and species (mice cycle in waves, whereas humans cycle in a mosaic pattern) ${ }^{1}$. The hypodermal fat layer in the skin changes thickness through the cycle. When thinnest, during the telogen phase ${ }^{40}$, this is often reported as an abnormal phenotype. Sebaceous gland size also changes through the hair cycle, making estimation of the size of this gland an unpredictable feature that is also commonly misinterpreted ${ }^{41}$. Changes in the numbers of hair follicles can be misinterpreted owing to artifacts of section orientation (Fig. 1) $)^{42}$.

Male mice have modified sebaceous glands (with a large excretory duct along the penis) known as preputial glands; these 


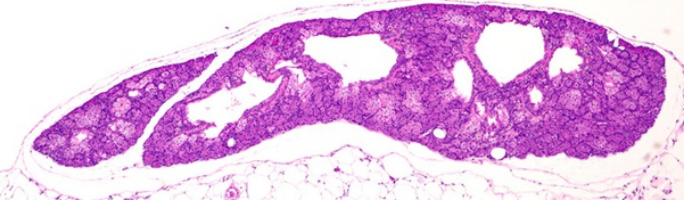

FIGURE 2 | Normal mouse preputial gland showing glandular tissue with central ducts. Two publications reported normal glands as teratomas or carcinomas. Hematoxylin and eosin stain, $4 \times$ magnification.

are also known as clitoral glands in the inguinal area of females (Fig. 2). These tissues have been diagnosed as teratomas or skin tumors ${ }^{33,43,44}$, and an erratum has been published for one of the publications in question ${ }^{43}$. Mouse accessory sex glands include various prostate lobes, seminal vesicles and other structures, the architecture of which differs from that of humans. Tissue artifacts have been diagnosed as early stage prostate cancer ${ }^{45}$ (Fig. 3).

Immunohistochemistry findings can also be problematic in publications with pathology results. Owing to omission of proper controls, authors often report positive labeling (often with a brown chromogen) of cells and tissues that appear to represent nonspecific background staining ${ }^{46}$. A good example was reported in mouse prostate epithelial cells and connective tissue ${ }^{47}$ using an anti-human antibody that has never been shown (even by the company selling the antibody) to work in mouse tissues. Mast cells are often nonspecifically positive in mouse tissues using peroxidase-based reagents ${ }^{48}$.

\section{When is a neoplasm not a neoplasm?}

Knowledge of appropriate nomenclature is also important for accurate reporting. Neoplasms and their preneoplastic/precancerous lesions are commonly found in mouse experiments involving chemical carcinogens and/or in genetically engineered mice. Many papers involving mice with these induced or spontaneous lesions do not refer to the publications that focus on standardized nomenclature for the organ or disease under investigation, such as those noted above and in our reference list. Although many journals list in their 'instructions for authors' that they require authors to use

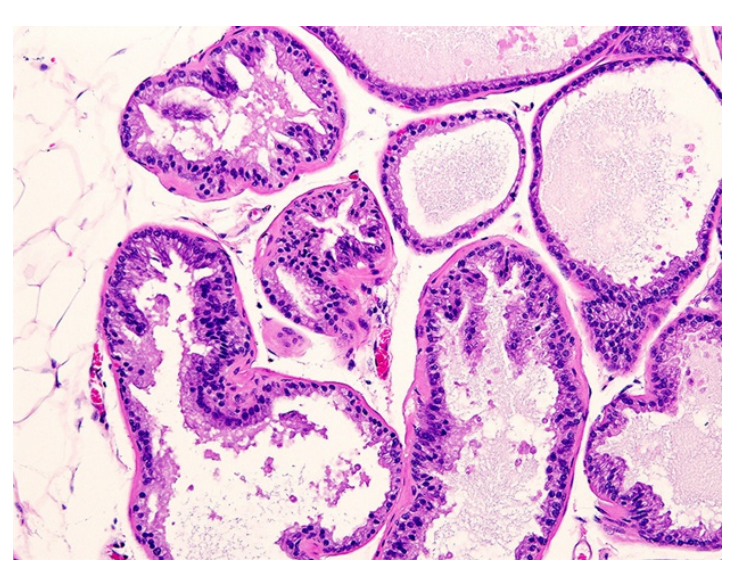

FIGURE 3 | Normal prostate of young mouse with artifactual folds of the acinar epithelium that were misdiagnosed as early stage prostate cancer in a publication. Hematoxylin and eosin stain, 40x magnification.

\begin{tabular}{|c|c|c|}
\hline Genetic location & Symbol & Why matched? \\
\hline Chr4 $82.89 \mathrm{~cm}$ & $\begin{array}{l}\text { Tnfrsf25, tumor } \\
\text { necrosis factor receptor } \\
\text { superfamily, member } 25\end{array}$ & synonym: TRAMP \\
\hline Chr1 4.18 cM & $\begin{array}{l}\text { Tram1, translocating } \\
\text { chain-associating } \\
\text { membrane protein } 1\end{array}$ & synonym: TRAMP \\
\hline Chr Unknown & $\begin{array}{l}\mathrm{Tg}(\mathrm{TRAMP}) 8247 \mathrm{Ng}, \\
\text { transgene insertion } 8247, \\
\text { Norman M Greenberg }\end{array}$ & $\begin{array}{l}\text { currentSymbol: } \\
\text { Tg(TRAMP)8247 Ng }\end{array}$ \\
\hline Chr1 $72.12 \mathrm{~cm}$ & Dpt, dermatopontin & humanSynonym: TRAMP \\
\hline
\end{tabular}

standardized nomenclature, this requirement is often not enforced by editors. This policy holds not only for diagnostic terms, but also for mouse strain and allele designations, as mouse genetic nomenclature is very uniformly standardized ${ }^{49,50}$. Some examples of questionable diagnoses of preneoplastic and neoplastic lesions of mice are given below.

The most widely used prostate cancer mouse model, commonly called TRAMP, is an example of the misuse of standardized nomenclature. A search of Mouse Genome Informatics (http://www.informatics. jax.org/genes.shtml) yielded four matches (Table 2), only one of which is the transgenic line used for prostate cancer research: $\operatorname{Tg}$ (TRAMP) $08247 \mathrm{Ng}^{51}$. With over 600 publications, this transgenic line is often considered to be the best mouse model of human prostate cancer. $\operatorname{The} \operatorname{Tg}$ (TRAMP) $08247 \mathrm{Ng}$ line was noted to have a high incidence of prostate adenocarcinoma metastases, suggesting that this was a good model for human prostate cancer. However, these were later shown to be of neuroendocrine origin rather than epithelial, with non-neuroendocrine epithelial being the most common in humans (Fig. 4) ${ }^{51-53}$. Phylloides prostate carcinomas, initially diagnosed in the first $\operatorname{Tg}$ (TRAMP) $08247 \mathrm{Ng}$ publication,

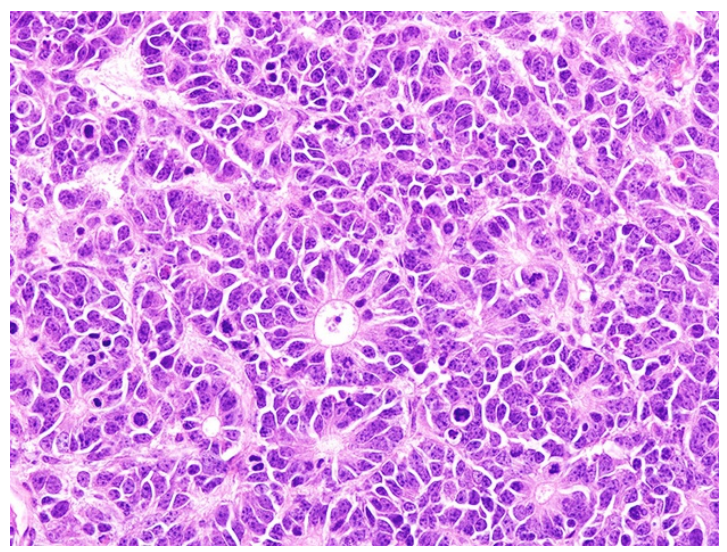

FIGURE 4 | Neuroendocrine carcinoma in the prostate of a TRAMP $(\mathrm{Tg}(\mathrm{TRAMP}) 8247 \mathrm{Ng})$ mouse. These mice were reported to develop highly metastatic prostate adenocarcinoma. Hematoxylin and eosin stain, 40x magnification. 


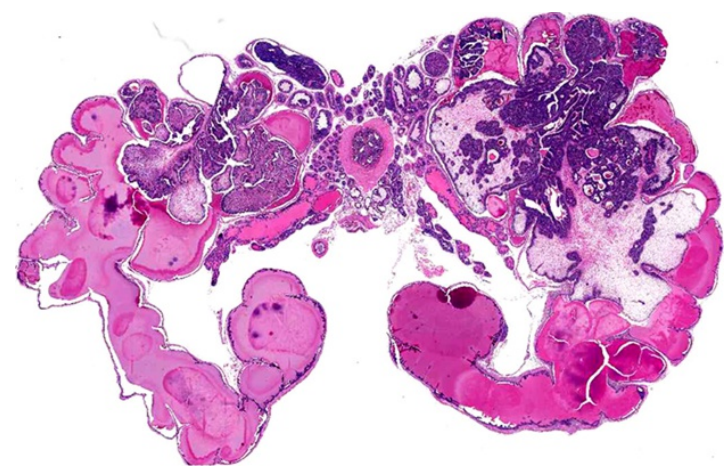

FIGURE 5 | Benign (epithelial-stromal) tumor in the seminal vesicles of a TRAMP mouse. Note neoplastic growth into the lumen and no invasion. These lesions were reported as phylloides prostate carcinomas. Hematoxylin and eosin stain, $4 \times$ magnification.

were later shown to be adenomas or benign epithelial-stromal tumors of the seminal vesicles (Fig. 5) ${ }^{51,54}$. Metastatic prostate carcinoma to bone marrow was described in a new genetically engineered mouse model, but a pathology nomenclature consensus committee determined that the cases were merely direct invasion from a large prostate mass ${ }^{51,55}$.

Inflammatory lesions caused by bacteria have sometimes been reported as tumors (neoplasms) ${ }^{56}$, as have been other types of inflammation ${ }^{57}$. This may be technically correct, as some textbooks define tumor literally as any type of swelling and one of the five cardinal signs of inflammation, but inflammation should and can be easily differentiated from neoplasia. Lymphomas and leukemias are often difficult to diagnose accurately. Tail tumors in transgenic mice were reported to be large granular lymphocytic (LGL) leukemia ${ }^{58}$, but other investigators working with the same mice found sarcomas of various types, including those arising in tendons and nerves in the tail (Figs. 6-8). These tail tumors may have developed accompanying inflammatory responses that included LGLs. Large spleens have been diagnosed as myeloproliferative disorders and leukemias, especially in mice with ulcerative skin

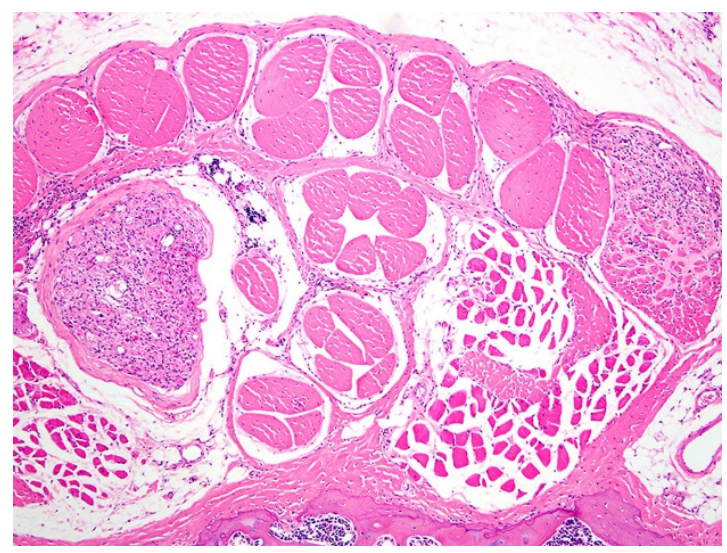

FIGURE 6 | Tail of a HTLV-I tax transgenic mouse with early neoplasms (on left and right side) of tendon origin. This mouse was reported to develop leukemia and not tendon neoplasms. Hematoxylin and eosin stain, $4 \times$ magnification.

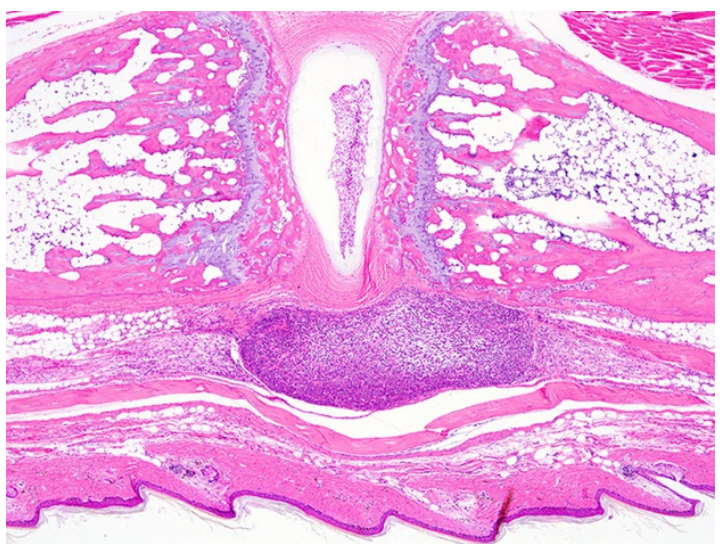

FIGURE 7 | Tail of a HTLV-I tax transgenic mouse with an early tumor of tail tendon origin (darker tumor in the lower portion of the figure beneath the skin). This mouse was reported to develop leukemia. Hematoxylin and eosin stain, $4 \times$ magnification.

lesions that cause reactive myeloid hyperplasia in the spleen ${ }^{59}$. Using a Helicobacter felis mouse gastric model, a research group developed a model of chronic gastritis that eventually was reported to develop gastric lymphomas. These changes appeared histologically unconvincing as described in the initial publication. In this case, however, a subsequent publication did provide molecular proof that these were indeed lymphomas ${ }^{60}$.

\section{How to increase reproducibility by improving nomenclature usage}

Problems in pathology evaluation may occur at various stages of the study, leading to questionable pathology interpretation in the submitted or published manuscript. To increase reproducibility in mouse studies, a trained pathologist knowledgeable in rodent pathology nomenclature should be involved, in study design, manuscript writing and/or chosen by the editors during the peer-review process. Pathology nomenclature in the paper should follow general guidelines for mouse pathology, as published by international committees and experts.

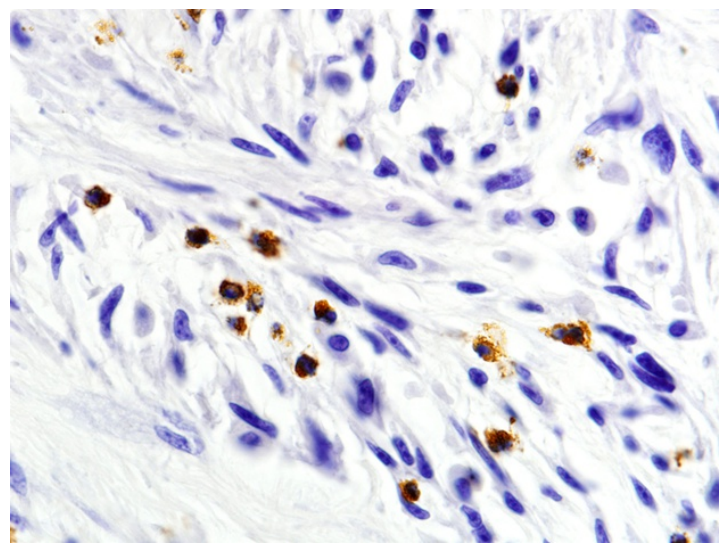

FIGURE 8 | Early tail tendon sarcoma showing tumor infiltration by myeloperoxidase-positive neutrophils. Immunoperoxidase, 40x magnification. 
As discussed above, the ability of a pathologist to accurately diagnose lesions in laboratory animals, especially rodents, depends on training and experience. Experience includes the use of widely acceptable veterinary pathology and speciesspecific nomenclature that is often provided through publications by expert groups of pathologists and by international or national committees ${ }^{33,61-65}$ and in books with multiple authors ${ }^{61,66-69}$. Others have proposed formal ontologies for data capture and analysis ${ }^{39,70,71}$, which are also based on international nomenclatures and informatics standards.

There are numerous publications on neoplastic diseases in mice, especially in genetically engineered mice. The National Cancer Institute Mouse Models of Human Cancer Consortium has established pathology committees over the past 15 years to develop nomenclatures for several important organs $33,51,61$. The publications on neoplastic diseases in mammary gland, prostate, lung, intestine, brain, skin and pancreas provide important guidelines for investigators. The INHAND pathology nomenclatures have similarly created detailed terminological recommendations for both proliferative and non-proliferative lesions under the auspices of the committees established by a consortium of societies of toxicologic pathology ${ }^{63}$. These, together with detailed publications on individual classes of lesion, make up a substantial terminological corpus with which pathologists making diagnoses should be familiar.

\section{Conclusion}

Compared with factors confounding the reproducibility of mouse biological experiments originating in experimental design, husbandry and microbiome, the problem of reliable histopathological interpretation of experimental animals is perhaps one of the most tractable sources of error. Enrollment of an experienced pathologist onto a study early in its inception and planning stages and then its subsequent analysis is clearly highly desirable, as many of the problems we discussed above are unlikely to arise under the guidance of appropriately trained personnel. The issue of finding experienced mouse pathologists has been discussed at length elsewhere ${ }^{2,24,34}$, although the authors feel that the mouse pathology community is sufficiently interactive that good advice can easily be sought out by motivated investigators.

Changes in priorities at journals and funding agencies are also needed to substantially improve the reliability of pathology in mouse model studies. The availability of the primary images on which experimental conclusions are based should be mandatory at journals and consistent with the FAIR guidelines ${ }^{5,72}$. Similarly, funding agencies need to pay more attention to the intended use of histopathology in grant applications and insist on provision of appropriate expertise with an appropriate budget. Increased stringency surrounding the processes of funding and publishing studies might represent more effort for researchers, reviewers and journal editors, but will reduce the instances of flawed histopathology that we see in many journals today ${ }^{73}$.

\section{ACKNOWLEDGMENTS}

This work was supported in part by US National Institutes of Health grants R01 AR049288, CA089713 and R21 AR063781 (to J.P.S.) and by The Warden and Fellows of Robinson College, Cambridge (to P.N.S.).

\section{COMPETING FINANCIAL INTERESTS}

J.P.S. has a research contract with BIOCON LLC for preclinical trials that does not involve any of the issues raised in this article.

\section{Received 17 October 2016; accepted 19 December 2016}

Published online at http://www.nature.com/laban

1. Sundberg, J.P., Nanney, L.B., Fleckman, P. \& King, L.E. Skin and adnexa in Comparative Anatomy and Histology (eds. P.M. Treuting \& S.M. Dintzis) 433-455 (Elsevier, Amsterdam, 2012).

2. Treuting, P.M. et al. The vital role of pathology in improving reproducibility and translational relevance of aging studies in rodents. Vet. Pathol. 53, 244-249 (2016).

3. Scudamore, C.L. et al. Recommendations for minimum information for publication of experimental pathology data: MINPEPA guidelines. $J$. Pathol. 238, 359-367 (2016).

4. Sundberg, J.P. et al. Approaches to investigating complex genetic traits in a large-scale inbred mouse aging study. Vet. Pathol. 53, 456-467 (2016).

5. Schofield, P.N., Ward, J.M. \& Sundberg, J.P. Show and tell: disclosure and data sharing in experimental pathology. Dis. Model. Mech. 9, 601-605 (2016).

6. Begley, C.G. \& Ellis, L.M. Drug development: raise standards for preclinical cancer research. Nature 483, 531-533 (2012).

7. Begley, C.G. \& Ioannidis, J.P. Reproducibility in science: improving the standard for basic and preclinical research. Circ. Res. 116, 116-126 (2015).

8. Prinz, F., Schlange, T. \& Asadullah, K. Believe it or not: how much can we rely on published data on potential drug targets? Nat. Rev. Drug Discov. 10, 712 (2011).

9. Wadman, M. NIH mulls rules for validating key results. Nature $500,14-16$ (2013).

10. Baker, M. 1,500 scientists lift the lid on reproducibility. Nature 533, 452-454 (2016)

11. Collins, F.S. \& Tabak, L.A. Policy: NIH plans to enhance reproducibility. Nature 505, 612-613 (2014).

12. Drucker, D.J. Never waste a good crisis: confronting reproducibility in translational research. Cell Metab. 24, 348-360 (2016).

13. Justice, M.J. \& Dhillon, P. Using the mouse to model human disease: increasing validity and reproducibility. Dis. Model. Mech. 9, 101-103 (2016).

14. Anonymous. Troublesome variability in mouse studies. Nat. Neurosci. 12, 1075 (2009).

15. Baker, M. Dutch agency launches first grants programme dedicated to replication. Nature doi:10.1038/nature.2016.20287 (2016).

16. Frye, S.V. et al. Tackling reproducibility in academic preclinical drug discovery. Nat. Rev. Drug Discov. 14, 733-734 (2015).

17. McNutt, M. Journals unite for reproducibility. Science 346, 679 (2014).

18. Reardon, S. A mouse's house may ruin experiments. Nature 530, 264 (2016).

19. Servick, K. Of mice and microbes. Science 353, 741-743 (2016).

20. Stappenbeck, T.S. \& Virgin, H.W. Accounting for reciprocal hostmicrobiome interactions in experimental science. Nature 534, 191-199 (2016).

21. Morrison, S.J. Time to do something about reproducibility. elife 3, 03981 (2014).

22. Maher, B. Cancer reproducibility project scales back ambitions. Nature doi:10.1038/nature.2015.18938 (2015).

23. Ward, A., Baldwin, T.0. \& Antin, P.B. Research data: silver lining to irreproducibility. Nature 532, 177 (2016).

24. Sundberg, J.P., Hogenesch, H., Nikitin, A.Y., Treuting, P.M. \& Ward, J.M. Training mouse pathologists: ten years of workshops on the pathology of mouse models of human disease. Toxicol. Pathol. 40, 823-825 (2012).

25. Grenz, A. et al. Equilibrative nucleoside transporter 1 (ENT1) regulates post-ischemic blood flow during acute kidney injury in mice. J. Clin. Invest. 122, 693-710 (2012).

26. Chaudhry, A. et al. CD4+ regulatory T cells control TH17 responses in a Stat3-dependent manner. Science 326, 986-991 (2009).

27. Frith, C.H. \& Ward, J.M. Color Atlas of Neoplastic and Non-neoplastic Lesions in Aging Mice (Elsevier, Amsterdam, 1988).

28. Brunnert, S.R., Shi, S. \& Chang, B. Chromosomal localization of the loci responsible for dystrophic cardiac calcinosis in DBA/2 mice. Genomics 59, 105-107 (1999). 
29. Ivandic, B.T. et al. New Dyscalc loci for myocardial cell necrosis and calcification (dystrophic cardiac calcinosis) in mice. Physiol. Genomics 6, 137-144 (2001).

30. Li, Q. et al. Mouse genome-wide association study identifies polymorphisms on chromosomes 4, 11 and 15 for age-related cardiac fibrosis. Mamm. Genome 27, 179-190 (2016).

31. Valenzuela, N. et al. Cardiomyocyte-specific conditional knockout of the histone chaperone HIRA in mice results in hypertrophy, sarcolemmal damage and focal replacement fibrosis. Dis. Model. Mech. 9, 335-345 (2016).

32. Ince, T.A. et al. Do-it-yourself (DIY) pathology. Nat. Biotechnol. 26, 978-979 (2008).

33. Cardiff, R.D., Ward, J.M. \& Barthold, S.W. 'One medicine-one pathology': are veterinary and human pathology prepared? Lab. Invest. 88, 18-26 (2008).

34. Barthold, S.W. et al. From whence will they come? A perspective on the acute shortage of pathologists in biomedical research. J. Vet. Diagn. Invest. 19, 455-456 (2007).

35. Bult, C.J. et al. Mouse Tumor Biology (MTB): a database of mouse models for human cancer. Nucleic Acids Res. 43, D818-D824 (2015).

36. Krupke, D.M., Begley, D.A., Sundberg, J.P., Bult, C.J. \& Eppig, J.T. The Mouse Tumor Biology database. Nat. Rev. Cancer 8, 459-465 (2008).

37. Hill, D.P. et al. The mouse Gene Expression Database (GXD): updates and enhancements. Nucleic Acids Res. 32, D568-D571 (2004).

38. Schofield, P.N. et al. Pathbase: a new reference resource and database for laboratory mouse pathology. Radiat. Prot. Dosimetry 112, 525-528 (2004)

39. Schofield, P.N., Gruenberger, M. \& Sundberg, J.P. Pathbase and the MPATH ontology. Community resources for mouse histopathology. Vet. Pathol. 47, 1016-1020 (2010).

40. Chase, H.B., Montagna, W. \& Malone, J.D. Changes in the skin in relation to the hair growth cycle. Anat. Rec. 116, 75-81 (1953).

41. Levkovich, T. et al. Probiotic bacteria induce a 'glow of health'. PLOS ONE 8, e53867 (2013).

42. Neutelings, T.e.a. Skin physiology in microgravity: a 3-month stay aboard ISS induces dermal atrophy and affects cutaneous muscle and hair follicles cycling in mice. npj Microgravity 1, 15002 (2015).

43. Fu, L., Pelicano, H., Liu, J., Huang, P. \& Lee, C. The circadian gene Period2 plays an important role in tumor suppression and DNA damage response in vivo. Cell 111, 41-50 (2002).

44. Nakamura, Y. et al. Phospholipase Cdelta1 is required for skin stem cell lineage commitment. EMBO J. 22, 2981-2991 (2003).

45. Maddison, L.A., Sutherland, B.W., Barrios, R.J. \& Greenberg, N.M. Conditional deletion of Rb causes early stage prostate cancer. Cancer Res. 64, 6018-6025 (2004).

46. Ward, J.M. \& Rehg, J.E. Rodent immunohistochemistry: pitfalls and troubleshooting. Vet. Pathol. 51, 88-101 (2014).

47. Mirantes, C. et al. An inducible knockout mouse to model the cellautonomous role of PTEN in initiating endometrial, prostate and thyroid neoplasias. Dis. Model. Mech. 6, 710-720 (2013).

48. Bolon, B. \& Calderwood-Mays, M.B. Conjugated avidin-peroxidase as a stain for mast cell tumor. Vet. Pathol. 25, 523-525 (1988).

49. Sundberg, J.P. \& Schofield, P.N. A mouse by any other name. J. Invest. Dermatol. 129, 1599-1601 (2009).

50. Sundberg, J.P. \& Schofield, P.N. Commentary: mouse genetic nomenclature. Standardization of strain, gene, and protein symbols. Vet. Pathol. 47, 1100-1104 (2010).

51. Ittmann, M. et al. Animal models of human prostate cancer: the consensus report of the New York meeting of the Mouse Models of Human Cancers Consortium Prostate Pathology Committee. Cancer Res. 73, 2718-2736 (2013).
52. Chiaverotti, T. et al. Dissociation of epithelial and neuroendocrine carcinoma lineages in the transgenic adenocarcinoma of mouse prostate model of prostate cancer. Am. J. Pathol. 172, 236-246 (2008).

53. Suttie, A.W. et al. An investigation of the effects of late-onset dietary restriction on prostate cancer development in the TRAMP mouse. Toxicol. Pathol. 33, 386-397 (2005).

54. Tani, Y., Suttie, A., Flake, G.P., Nyska, A. \& Maronpot, R.R. Epithelialstromal tumor of the seminal vesicles in the transgenic adenocarcinoma mouse prostate model. Vet. Pathol. 42, 306-314 (2005).

55. Ding, Z. et al. Telomerase reactivation following telomere dysfunction yields murine prostate tumors with bone metastases. Cell 148, 896-907 (2012).

56. Fukumoto, S. et al. Ameloblastin is a cell adhesion molecule required for maintaining the differentiation state of ameloblasts. J. Cell Biol. 167, 973-983 (2004).

57. Zhao, Z.G. et al. MicroRNA profile of tumorigenic cells during carcinogenesis of lung adenocarcinoma. J. Cell. Biochem. 116, 458-466 (2015).

58. Grossman, W.J. et al. Development of leukemia in mice transgenic for the tax gene of human T-cell leukemia virus type I. Proc. Natl. Acad. Sci. USA 92, 1057-1061 (1995).

59. Wheeler, D.L. et al. Overexpression of protein kinase $C-\varepsilon$ in the mouse epidermis leads to a spontaneous myeloproliferative-like disease. Am. J. Pathol. 166, 117-126 (2005).

60. Mueller, A. et al. The role of antigenic drive and tumor-infiltrating accessory cells in the pathogenesis of helicobacter-induced mucosaassociated lymphoid tissue lymphoma. Am. J. Pathol. 167, 797-812 (2005).

61. Cardiff, R.D. et al. Precancer in mice: animal models used to understand, prevent, and treat human precancers. Toxicol. Pathol. 34, 699-707 (2006).

62. Cesta, M.F. et al. The National Toxicology Program Web-based nonneoplastic lesion atlas: a global toxicology and pathology resource. Toxicol. Pathol. 42, 458-460 (2014).

63. Mann, P.C. et al. International harmonization of toxicologic pathology nomenclature: an overview and review of basic principles. Toxicol. Pathol. 40, 7S-13S (2012).

64. Mohr, U. International Classification of Rodent Tumors: The Mouse (Springer, Berlin, 2001).

65. Nolte, T. et al. Nonproliferative and proliferative lesions of the gastrointestinal tract, pancreas and salivary glands of the rat and mouse. J. Toxicol. Pathol. 29, 1S-125S (2016).

66. Jones, T.C., Dungworth, D.L. \& Mohr, U. Respiratory System (SpringerVerlag, Berlin, 1996).

67. Maronpot, R.R. Pathology of the Mouse (Cache River Press, Vienna, Illinois, 1999).

68. Mohr, U. et al. Pathobiology of the Aging Mouse (ILSI Press, Washington, D.C., 1996).

69. Ward, J.M., Mahler, J.F., Maronpot, R.R. \& Sundberg, J.P. (eds.). Pathology of Genetically Engineered Mice (Iowa State University Press, 2000).

70. Schofield, P.N., Sundberg, J.P., Sundberg, B.A., McKerlie, C. \& Gkoutos, G.V. The mouse pathology ontology, MPATH; structure and applications. J. Biomed. Semantics 4, 18 (2013).

71. Fisher, H.M. et al. Derm0; an ontology for the description of dermatologic disease. J. Biomed. Semantics 7, 38 (2016).

72. Wilkinson, M.D. et al. The FAIR Guiding Principles for scientific data management and stewardship. Sci. Data 3, 160018 (2016).

73. Wolf, J.C. \& Maack, G. Evaluating the credibility of histopathology data in environmental endocrine toxicity studies. Environ. Toxicol. Chem. doi:10.1002/etc.3695 (2017). 